\title{
Navigating the Space: Evaluating a 3D-Input Device in Placement and Docking Tasks
}

\author{
Elke Mattheiss \\ CURE Center for Usability \\ Research \& Engineering \\ Modecenterstraße 17 / Objekt 2 \\ 1110 Wien \\ mattheiss@cure.at
}

\author{
Johann Schrammel \\ CURE Center for Usability \\ Research \& Engineering \\ Modecenterstraße 17 / Objekt 2 \\ 1110 Wien \\ schrammel@cure.at
}

\author{
Manfred Tscheligi \\ ICT\&S, University \\ of Salzburg \\ S.-Haffner-Gasse 18 \\ 5020 Salzburg \\ manfred.tscheligi@sbg.ac.at
}

\begin{abstract}
We present a study investigating the performance in a 3D object manipulation task with a mouse and a dedicated input device (SpaceNavigator). Previous research delivered ambiguous results about the performance in different 3D tasks. Therefore we used placement (only translation) as well as docking (translation and rotation) tasks. Twelve participants experienced with 3D software took part in the study. They had to translate and rotate 30 cubes with the mouse and the SpaceNavigator (altogether 60 tasks) to place them on a chessboard in Autodesk Maya. The results show an outperformance of the mouse over the SpaceNavigator in the placement tasks but not in the docking tasks, which require a higher extent of object manipulation. Although the SpaceNavigator did not outperform the mouse, considering the number of tasks with the SpaceNavigator and further results of the study (like the learning effect and subjective feedback), the usage of a higher degree-of-freedom device for tasks with multiple simultaneous object manipulations seems reasonable.
\end{abstract}

Input device. 3D interaction. Object manipulation. Empirical Evaluation. SpaceNavigator.

\section{INTRODUCTION}

The manipulation of three dimensional (3D) objects is a task frequently needed in different computer applications, especially in the context of computeraided design (CAD). In a typical desktop computing environment the user however has no direct access to the 3D-object, and it has to be controlled and manipulated using interaction mechanisms with a limited number of degrees of freedom (DoFs). Much previous research was committed to find good ways of overcoming this problem. A considerable part of this research concentrated on designing new 3D input devices, to enable the user to intuitively control three to six DoFs simultaneously. Despite these efforts the mouse remains the most widely used input device for 3D tasks, and frequently outperforms dedicated 3D input devices in empirical evaluation studies (e.g. Bérard et al., 2009). Besides these research efforts also first commercial products became available, e.g. SpaceNavigator ${ }^{1}$. It however remains questionable whether these devices can actually deliver the advertised gains in performance in $3 \mathrm{D}$ interaction.

The present study aims to evaluate the performance of an existing commercial 3D-

\footnotetext{
${ }^{1}$ http://www.3dconnexion.com/
}

controller (SpaceNavigator) in comparison to mouse-only control, to study the learning effects, and the influence of possible moderating factors (task type) in the use of different input devices.

\section{RELATED WORK}

\subsection{D object manipulation}

A typical task in 3D environments is the manipulation of virtual objects which includes actions like selecting, scaling, rotating, translating, deleting and editing (Hand, 1997). Whereas target selection in a 3D scene can be simplified to a two DoF task, placement and rotation can be considered as true 3D tasks. The simplest way to deal with 3D tasks with a two DoF input device (like the conventional mouse) is to decompose a manipulation task into separate actions (e.g. positioning and orientation). Unfortunately, there is no intuitive mapping of these tasks with more than two DoFs to the usage of a mouse with sliders, menus, or buttons. To overcome these constraints and provide most unambiguous mappings of the 2D cursor motion to a specific object manipulation as possible, several approaches of manipulation techniques have been developed. Selected examples for such manipulation techniques are 
skitters and jacks (Bier, 1986), object associations (Bukowski \& Séquin, 1995), using constraints to restrict object motion in 3D scene (Smitz, Salzman \& Stuerzlinger, 2001), and semantic 3D picking (Elmqvist \& Fekete, 2008).

\subsection{Dedicated input devices for 3D}

Another approach for improving 3D interaction is to design input devices particularly adapted to the needs of performing $3 \mathrm{D}$ tasks. Examples for such 3D input devices are research prototypes like the Rockin'Mouse (Balakrishnan et al., 1997) and the GlobeFish and GlobeMouse (Froehlich et al., 2006) and commercial products like the SpaceNavigator. The crucial advantage of $3 \mathrm{D}$ input devices is that they enable the users to accomplish a true 3D task with one single action instead of dividing it into two or more actions (i.e. translating and rotating an object simultaneously). Allowing the control of six DoFs at the same time should increase the users' performance in 3D tasks. However the conventional mouse outperforms 3D input devices in various research studies. A recent study from Bérard and colleagues (2009) for example showed a clear outperformance of the mouse over three different 3D input devices, including the SpaceNavigator. The authors used placement tasks in their study, because they considered it as the most fundamental and frequent task in 3D interaction. In contrast Hinckley and colleagues (1997) as well as McMahan and colleagues (2006) found an outperformance of six DoF devices for docking and respectively rotation tasks.

The outperformance of the mouse is traced back by Teather and Stuerzlinger (2008) to the users' familiarity with the mouse, the reduced dimensionality of a task and the supporting surface required when accomplishing a 3D task with the mouse. Another factor may be that users do not conduct translations and rotations at the same time but successively with a six DoF input device (Masliah \& Milgram, 2000).

After all no clear preference for one or the other input device can be stated. Therefore the present study focuses on the performance with input device in placement as well as docking tasks, to investigate the research question: Does the task type influence with which kind of input device users perform 3D interaction tasks better?

\section{METHOD}

To answer our research question we invited 12 participants to perform different placement and docking tasks using mouse and SpaceNavigator. Task completion times and a subjective usability rating were recorded. The following section describes the used method and materials in detail.

\subsection{Hypothesis}

To investigate the performance of the input devices we differentiated between placement (only translation) and docking (translation and rotation) tasks and between two phases of the trial progress (first 12 tasks of the testing trial versus last 12 tasks). Based on previous research the following hypotheses underlie the present study:

(i) Placement tasks: The participants' task completion time is significantly shorter with the mouse than with the SpaceNavigator.

(ii) Docking tasks: The participants' task completion time is significantly shorter with the SpaceNavigator than with the mouse. This advantage of the SpaceNavigator is higher for docking tasks requiring more object rotation.

(iii) Trial progress I: The participants' task completion time is significantly shorter at the beginning of the trial than at the end.

(iv) Trial progress II: The decrease of the task completion time (i.e. the learning effect) is higher with the SpaceNavigator than with the mouse.

\subsection{Participants}

For the evaluation 12 participants, experienced with 3D or graphic software, were recruited. Participation was voluntary and rewarded with 40 Euros. Participants were all male, right-handed and aged between 18 and 38 (mean age $=26.17$, standard deviation $(S D)=5.17$ ). They were all students or post-graduates mainly engaged in a technical context. All participants had no prior experience with the SpaceNavigator.

\subsection{Equipment}

The tasks were performed with Autodesk Maya ${ }^{2}$; a software for 3D animation. As dedicated 3D input device the six DoF SpaceNavigator was used (see Figure 1), which is intended to be used in the nondominant hand along with the mouse in the dominant one. The movements of the knob of the SpaceNavigator are mapped to the movements of the respective 3D objects. The setup for the SpaceNavigator was the default setup i.e. pan right/left to move the object to the right/left, pull/push to move the object up/down, pan forward/backward to zoom in/out and tilt, spin and roll to rotate the object according to the three axes. To translate and rotate objects with the mouse in Autodesk Maya, participants had to activate the translation or rotation mode with a key press and manipulate the cube with the aid of arrows and circles (see Figure 2).

\footnotetext{
${ }^{2}$ http://www.autodesk.de/
} 


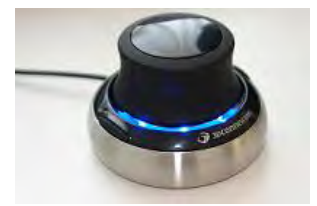

Figure 1: SpaceNavigator from 3Dconnexion

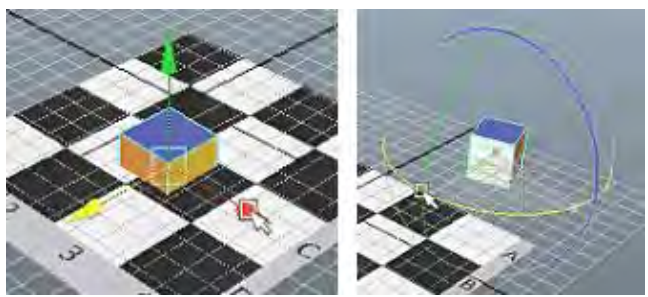

Figure 2: Translation (left side) and rotation (right side) of an object in Autodesk Maya with the aid of the mouse

\subsection{Procedure}

Each participant filled out a demographic questionnaire and was provided with a short instruction and training with the SpaceNavigator (three minutes). In this short training the participants tried out the SpaceNavigator by moving and rotating $3 \mathrm{D}$ objects.

For the actual trial participants had to place a cube with six differently coloured faces (three faces are visible at the beginning of the task) on a specific area of a chessboard (see Figure 3). To use tasks which are as similar as possible and only differ in the extent of rotation necessary to accomplish the task, the following four different types of tasks were included:

(i) Placement tasks: the cube had to be translated but not rotated;

(ii) Docking tasks: the cube had to be translated and rotated:

(a) Docking 1 - one visible face up: The cube had to be placed with a specific face up on a field - this face was one of the three faces visible at the beginning of the task;

(b) Docking 2 - one invisible face up: The cube had to be placed with a specific face up on a field - this face was one of the three faces invisible at the beginning of the task;

(c) Docking 3 - one face up and one face forward: The cube had to be placed with a specific face up and another specific face forward on a field - one of the faces was visible the other invisible at the beginning of the task.

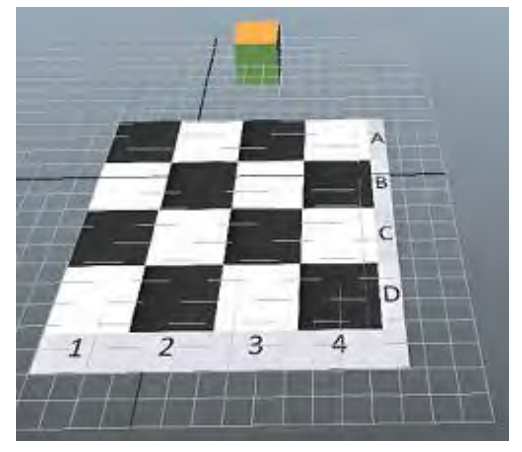

Figure 3: Task to place the cube (with a specific face) at a specific area of the chessboard

Altogether participants' performed 30 tasks with the mouse and the same 30 tasks with the SpaceNavigator. The ordering of the trials with the input devices was counterbalanced between participants (i.e. half of the participants started with the mouse, the other half with the SpaceNavigator). The task trial consisted of alternating placement and docking tasks, therefore each participant conducted 15 placement and five docking tasks of each of the three kinds (see Table 1). Thus the training comprises of five sets including six equivalent tasks (task 1-6, 7-12, 13-18, 19-24 and 25-30).

Following the tasks the participants' were surveyed regarding their subjective preference. Participants had to rate the usability of the input devices on scale from 1 (very good) to 5 (very bad). Furthermore they had to reason their answer.

Table 1: Ordering of the 30 tasks (first row) with altering placement $(P)$ and docking $(D 1, D 2, D 3)$ tasks - each six tasks combine into one task set

\begin{tabular}{|l|l|l|l|l|l|l|l|l|l|}
\hline 1 & 2 & 3 & 4 & 5 & 6 & 7 & $\ldots$ & 29 & 30 \\
\hline$P$ & $D 1$ & $P$ & $D 2$ & $P$ & $D 3$ & $P$ & $\ldots$ & $P$ & $D 3$ \\
\hline Task set 1 & & &
\end{tabular}

\subsection{Design}

To test the specified hypotheses related to placement and docking tasks two designs were

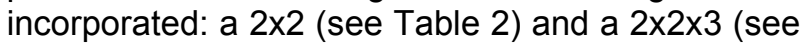
Table 3) within-subjects design. The independent variables were input device (mouse vs. SpaceNavigator), trial progress (begin $=$ task set 1 and 2 vs. end $=$ task set 4 and 5 ) and for the docking tasks also task type (docking 1,2 and 3 with increasing extent of required rotation). The dependent variable is the task completion time for the different tasks. For the 60 tasks in total participants needed about 50 minutes.

Table 2: 2x2 within-subjects design of placement tasks

\begin{tabular}{|c|c|c|c|}
\hline & \multicolumn{2}{|c|}{ Input device } \\
\hline & & Mouse & SpaceNavigator \\
\hline \multirow{2}{*}{$\begin{array}{c}\text { Trial } \\
\text { progress }\end{array}$} & begin & 12 & 12 \\
\hline & end & 12 & 12 \\
\hline
\end{tabular}


Table 3: $2 \times 2 \times 3$ within-subjects design of docking tasks

\begin{tabular}{|c|c|c|c|c|}
\hline & \multirow[b]{2}{*}{$\begin{array}{c}\text { Trial } \\
\text { progress }\end{array}$} & \multicolumn{2}{|c|}{ Input device } \\
\hline & & & Mouse & $\begin{array}{l}\text { SpaceNa } \\
\text { vigator }\end{array}$ \\
\hline \multirow{6}{*}{$\begin{array}{l}\text { Task } \\
\text { type }\end{array}$} & \multirow[t]{2}{*}{ Docking 1} & begin & 12 & 12 \\
\hline & & end & 12 & 12 \\
\hline & \multirow[t]{2}{*}{ Docking 2} & begin & 12 & 12 \\
\hline & & end & 12 & 12 \\
\hline & \multirow[t]{2}{*}{ Docking 3} & begin & 12 & 12 \\
\hline & & end & 12 & 12 \\
\hline
\end{tabular}

\section{RESULTS}

\subsection{Task completion times of the placement tasks}

To compare the participants' performance with the input devices according to pure placement tasks at the beginning and the end of the trial, a repeated measures two-way analysis of variance was calculated. The mean task completion time is significantly higher with the SpaceNavigator than with the mouse $\left(F_{1,11}=28.95, p=.000\right.$; Figure 4). Furthermore the task completion time at the beginning of the trial is significantly higher than at the end $\left(F_{1,11}=36.73, p=.000\right)$. Also the interaction between trial progress and input device is significant $\left(F_{1,11}=21.45, p=.001\right)$, which means that the trial progress shows a higher reduction of the task completion time for tasks conducted with the SpaceNavigator than with the mouse. The results of the analysis of variance were further explored by paired t-tests with alpha-corrections (due to multiple comparisons). They revealed that the task completion time with the mouse was significantly shorter than with the SpaceNavigator at the beginning $\left(t_{11}=-5.34, p=.000\right)$ as well as the end of the trial $\left(t_{11}=-4.58, p=.001\right)$.

\subsection{Task completion times of the docking tasks}

As three types of docking tasks - differing in the required extent of object rotation - were used, a further independent variable was incorporated in the design for docking tasks. A repeated measures three-way analysis of variance was calculated. Figure 5 shows a statistically significantly longer task completion time at the beginning (mean = $43.52, \mathrm{SD}=3.22)$ than at the end (mean $=33.54$, $S D=2.73)$ of the trial $\left(F_{1,11}=49.86, p=.000\right)$, but no difference in task completion time between the input devices $\left(F_{1,11}=0.41, p=.536\right)$. The interaction between trial progress and input device is not significant $\left(F_{1,11}=3.14, p=.104\right)$, but shows a small tendency that trial progress influences the performance with the input devices differently. The significant main effect of the docking task type $\left(F_{2,22}=7.80, p=.002\right)$ could not be interpreted because of the significant disordinal interaction between task type and input device $\left(F_{2,22}=11.00\right.$, $p=.000)$ and between task type and trial progress $\left(F_{2,22}=7.08, p=.004\right)$. Therefore simple effects tests were calculated for the different levels of the factors "trial progress" and "input device". The task completion times of the task types differs significantly at the beginning $\left(F_{2,22}=3.95, p=.034\right)$ as well as the end $\left(F_{2,22}=13.51, p=.000\right)$ of the trial.

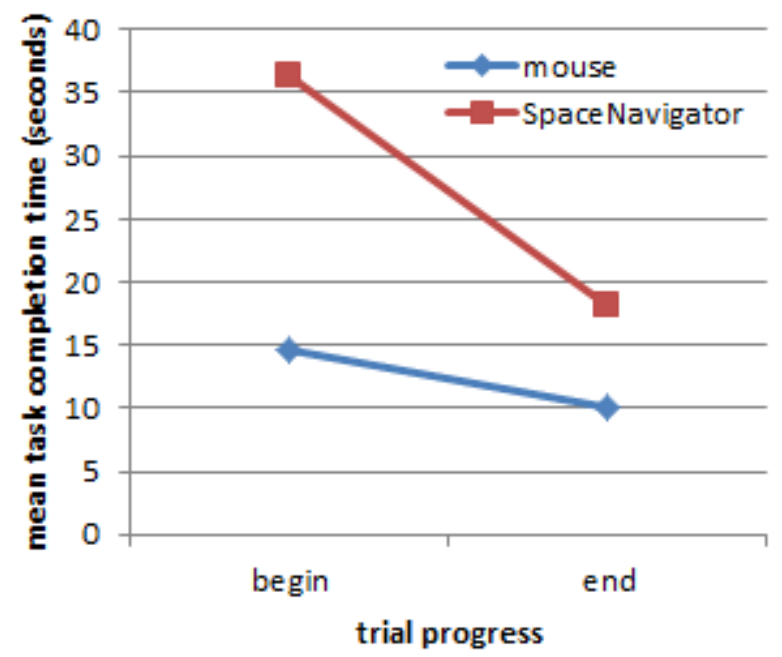

Figure 4: Mean task completion time for the placement tasks at the beginning and end of the task trial

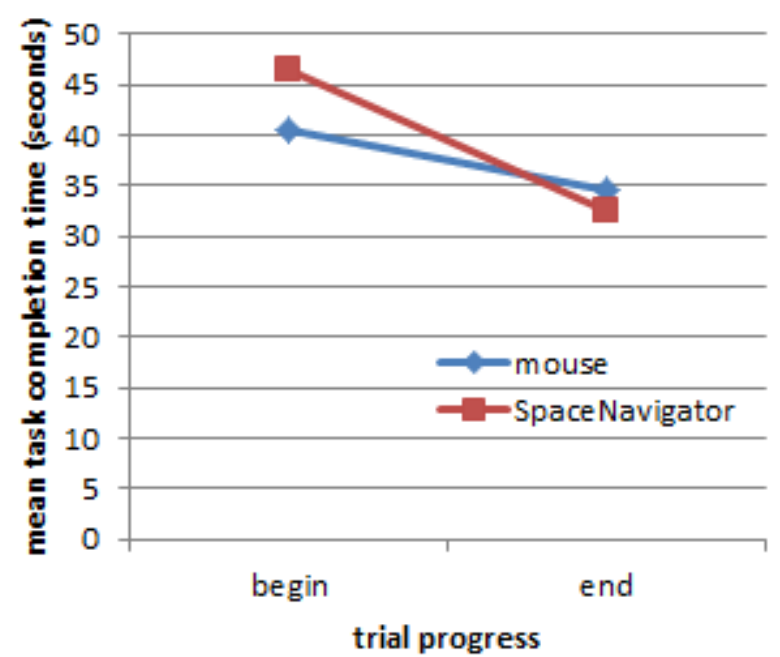

Figure 5: Mean task completion time for the docking tasks at the beginning and the end of the task trial

Using t-tests with Bonferroni corrected alpha-level reveals a significant difference between docking 2 and docking $3(p=.016)$ at the beginning of the trial, in terms of a higher task completion time for docking 3 (see Figure 6). At the end of the trial the task completion time of docking 1 is significantly lower than of docking $2(p=.026)$ and docking $3(p$ $=.001$ ). Considering the input device (see Figure 7) there was found a significant difference in the task completion time of the docking task types when using the mouse $\left(F_{2,22}=14.84, p=.000\right)$ but not when using the SpaceNavigator $\left(F_{2,22}=2.22, p=\right.$ 
.132). When using the mouse the task completion time for docking 3 was significantly higher than for docking $1(p=.002)$ and docking $2(p=.006)$.

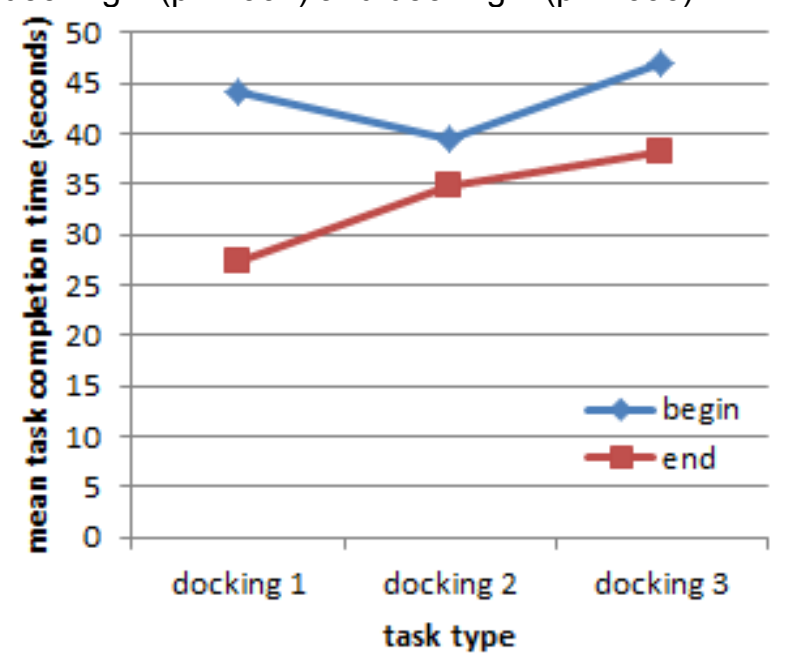

Figure 6: Mean task completion time for the docking tasks at the beginning and the end of the task trial

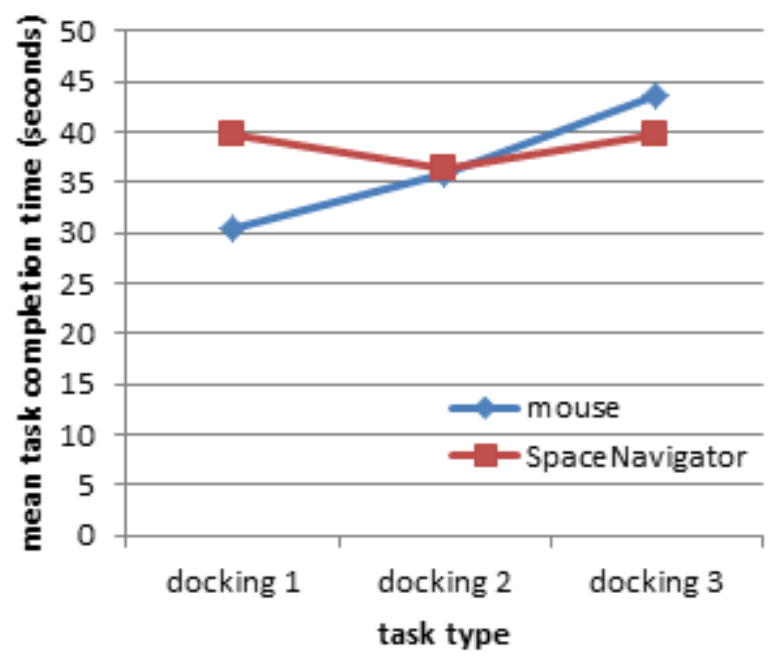

Figure 7: Mean task completion time for the docking tasks with the mouse and the SpaceNavigator

Three paired t-tests between mouse and SpaceNavigator for the three docking types showed that only in docking 1 tasks the mouse outperformed the SpaceNavigator $\left(\mathrm{t}_{11}=-3.73, \mathrm{p}=\right.$ $.003)$. For docking $2\left(t_{11}=-0.19, p=.85\right)$ and docking $3\left(t_{11}=0.96, p=.36\right)$ no significant difference between the input devices was found.

\subsection{Subjective measures}

Besides objective measures we also assessed the rating of the input devices. The average usability expressed on a scale from 1-good to 5-bad of the mouse is $2.5(S D=1.24)$, for the SpaceNavigator it is $1.58(\mathrm{SD}=0.67)$. Although there seems to be a tendency that participants rated the SpaceNavigator as more usable, the difference is not statistically significant with an alpha-level of .05 $\left(t_{11}=2.11, p=.060\right)$. From the participants' comments we learn that they clearly see the advantage of having the possibility to translate and rotate an object at the same time. They experience the SpaceNavigator as being fun, but also state that it is unfamiliar and requires some training.

\section{DISCUSSION}

\subsection{Input devices and task type}

The results of the present study suggest that the task type of a 3D interaction influences the efficiency of the used input devices.

For placement tasks which include the translation of the object in the 3D space, we found a clear superiority of the mouse over the SpaceNavigator in terms of the task completion time. This superiority decreases at the end of the trial suggesting a greater learning effect with the SpaceNavigator - but still remains significant. This result confirms our first hypothesis (task completion time in placement tasks is significantly shorter with the mouse than with the SpaceNavigator). Furthermore it is in line with the previous results of Bérard and colleagues (2009), that the mouse is more efficient for accurate placement in 3D interaction. This instance seems reasonable, since the advantage of a six DoF input device to enable translation and rotation simultaneously may not be relevant for a three DoF task. For such tasks the accurate two DoF mouse and an according support surface are perfectly adequate.

The situation is different with the docking tasks. Three different types of docking tasks were included in the study, differing in the extent of object rotation required to accomplish the task (docking 1 to 3 with increasing need for rotation). For this kind of tasks we did not find a general difference between the two input devices. Only for docking 1 tasks there was found a difference in terms of an outperformance of the mouse. This result does not confirm our second hypothesis (task completion time is significantly shorter with the SpaceNavigator than with the mouse for docking tasks) and is not in line with previous research (Hinckley et al., 1997; McMahan et al., 2006) reporting an outperformance of a six DoF device for docking and rotation tasks. A possible explanation for this result could be the amount of training. Because of the lack of prior experience with the SpaceNavigator, maybe 30 tasks with the SpaceNavigator were not enough to outperform the everyday used mouse. It is still to be clarified whether the small tendency that the task completion time decreases stronger between beginning and end of the trial for the SpaceNavigator than for the mouse (apparent in Figure 5) would continue if the number of tasks was increased. 
Also a more detailed consideration of the data reveals interesting insights. Whereas the performance for the task type docking 3 , which includes the highest level of rotation, was significantly lower than for the other two types when using the mouse, such a difference could not be found for the SpaceNavigator. This contradicts the results of Masliah and Milgram (2000), who found that users conduct rotations and translations separately instead of at the same time. The results of the present study suggests that with the SpaceNavigator the extent of required rotation is not as crucial for the task completion time as it is with the mouse - that is probably because translation and rotation can be done simultaneously.

\subsection{Trial progress}

Regarding the effect of the trial progress we compared the participants' performance in the first 12 tasks with the last 12 tasks. The results are like expected. The third hypothesis (task completion time is significantly shorter at the beginning of the trial than at the end) could be confirmed both the placement and the docking tasks. The fourth hypothesis (task completion time - i.e. the learning effect - is higher with the SpaceNavigator than with the mouse) could be confirmed only for the placement tasks - for the docking tasks only a small tendency was found.

\section{CONCLUSION}

We compared the performance of participants in placement and docking tasks with the two DoF mouse and the six DoF SpaceNavigator. The results of this comparison suggest that - at least in 30 conducted tasks - the higher DoF SpaceNavigator only pays off for tasks with a higher level of object manipulation (translation and rotation) but not for translation-only tasks. It is a matter of conjecture if the strong learning effect with the SpaceNavigator leaded to clear outperformance of the SpaceNavigator over the mouse, when the number of tasks would be increased. However considering the positive feedback of the participants about the SpaceNavigator it seems worthwhile that future research should focus on long-term observations including realistic tasks to clarify the actual advantages of six DoF input devices for 3D tasks.

\section{REFERENCES}

Balakrishnan, R., Baudel, T., Kurtenbach, G. and Fitzmaurice, G. (1997) The Rockin'Mouse: Integral 3d manipulation on a plane. In ACM Conference on Human Factors in Computing Systems (CHI'97), ACM (1997) 311-318.
Bérard, F., Ip, J., Benovoy, M., El-Shimy, D., Blum, J.R. and Cooperstock, J.R. (2009) Did "minority report" get it wrong? Superiority of the mouse over 3D input devices in a 3D placement task. In Proceedings of the IFIP TC International Conference on Human-Computer Interaction (INTERACT'09), 400-414, Berlin, Heidelberg, Springer.

Bier, E.A. (1986) Skitters and jacks: Interactive 3D positioning tools. In Proceedings of the 1986 Workshop on Interactive 3D Graphics, 183-196. ACM: New York, October 1986.

Bowman, D.A., Chen, J., Wingrave, C.A., Lucas, J., Ray, A., Polys, N.F., Li, Q., Haciahmetoglu, Y., Kim, J.-S., Kim, S., Boehringer, R. and Ni, T. (2006) New directions in 3D user interfaces. The International Journal of Virtual Reality, 5(2), 3-14.

Bukowski, R.W. and Sèquin, C.H. (1995) Object associations: A simple and practical approach to virtual 3D manipulation. In 1995 Symposium on Interactive 3D Graphics, 131-138, April 1995.

Elmqvist, N. and Fekete, J.-D. (2008) Semantic pointing for object picking in complex 3D environments. In Proceedings of Graphics Interface Conference, 243-250.

Froehlich, B., Hochstrate, J., Skuk, V. and Huckauf, A. (2006) The GlobeFish and the GlobeMouse: Two new six degree of freedom input devices for graphics applications. In ACM Conference on Human Factors in Computing Systems (CHI'06), ACM, 191-199.

Gittler, G. (1990) Dreidimensionaler Würfeltest (3DW): Ein Rasch-skalierter Test zur Messung des räumlichen Vorstellungsvermögens. Theoretische Grundlagen und Manual, Weinheim: Beltz Test.

Hand, C. (1997) A survey of 3D interaction techniques. Computer Graphics Forum, 16, 269281.

Hinckley, K., Tullio, J., Pausch, R., Proffitt, D. and Kassell, N. (1997) Usability analysis of 3D rotation techniques. In ACM Symposium on User Interface Software and Technology (UIST), ACM, 1-10.

Masliah, M.R. and Milgram, P. (2000) Measuring the allocation of control in a 6 degree-of-freedom docking experiment. In ACM Conference on Human Factors in Computing Systems (CHI'00), ACM, 25-32.

McMahan, R.P., Gorton, D., Gresock, J., McConnell, W. and Bowman, D.A. (2006) Separating the effects of level of immersion and 3D interaction techniques. In ACM Symposium on Virtual Reality Software and Technology, ACM, 108-111.

Smith, G., Salzman, T. and Stuerzlinger, W. (2001) 3D scene manipulation with 2D devices and constraints. In Graphics Interface 2001, 135-142, June 2001.

Teather, R.J. and Stuerzlinger, W. (2008) Assessing the effects of orientation and device on (constrained) 3D movement techniques. In IEEE Symposium on 3D User Interfaces, 43-50. 